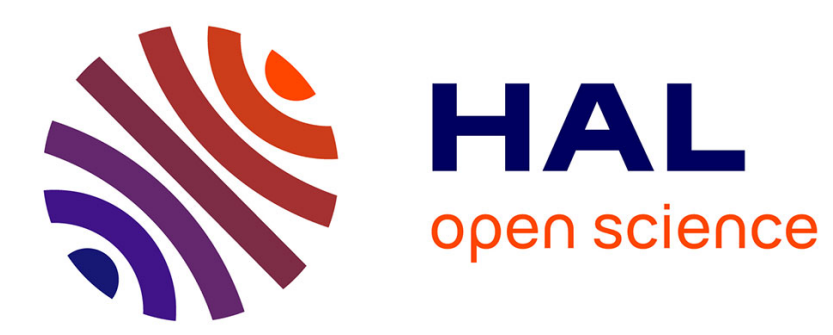

\title{
A LANDAU THEORY OF THE NAC POINT
}

\author{
Ludovic Benguigui
}

\section{To cite this version:}

Ludovic Benguigui. A LANDAU THEORY OF THE NAC POINT. Journal de Physique Colloques, 1979, 40 (C3), pp.C3-419-C3-421. 10.1051/jphyscol:1979384 . jpa-00218780

\section{HAL Id: jpa-00218780 https://hal.science/jpa-00218780}

Submitted on 1 Jan 1979

HAL is a multi-disciplinary open access archive for the deposit and dissemination of scientific research documents, whether they are published or not. The documents may come from teaching and research institutions in France or abroad, or from public or private research centers.
L'archive ouverte pluridisciplinaire HAL, est destinée au dépôt et à la diffusion de documents scientifiques de niveau recherche, publiés ou non, émanant des établissements d'enseignement et de recherche français ou étrangers, des laboratoires publics ou privés. 


\title{
A LANDAU THEORY OF THE NAC POINT
}

\author{
L. BENGUIGUI (*) \\ Laboratoire de Diélectriques, Faculté des Sciences Mirande, 21100 Dijon, France
}

\begin{abstract}
Résumé. - Bien que le point NAC où coexistent les phases nématique, smectique $A$ et smectique $C$, puisse être considéré comme un point de Lifshitz, nous avons pensé qu'il était utile de présenter une théorie du type Landau, avec deux paramètres d'ordre. On peut ainsi expliquer d'une manière simple le fait que la transition N-Sm C est toujours du premier ordre, comme il a été observé expérimentalement. On trouve aussi que la transition $\mathrm{Sm}$ A-Sm $\mathrm{C}$ peut être du premier ou du deuxième ordre suivant que cette transition est proche ou éloignée du point NAC. La topologie des diagrammes expérimentaux est en accord avec les résultats théoriques.
\end{abstract}

\begin{abstract}
Although the NAC point, where the three phases : nematic, smectic A and smectic C coexist, can be seen as a Lifshitz point, we think that it is interesting to propose a Landau theory with two order parameters. We are able to explain easily that the $\mathrm{N}-\mathrm{Sm} \mathrm{C}$ transition is always of first order, as experimentally observed. The Sm A-Sm C transition can be either first order or second order depending whether the transition point is near or far from the NAC point. The diagram topology we found is in agreement with the experimental results.
\end{abstract}

1. Introduction. - Recently Chen and Lubensky [1] have proposed an interesting application of the concept of Lifshitz point to the NAC point : a triple point where three transition lines (Nematic-Smectic A, Smectic A-Smectic C and Nematic-Smectic C) intersect in a phase diagram (for example temperature-composition). In their interpretation, the tilt angle of the $\mathrm{Sm} \mathrm{C}$ phase is not an order parameter, but it is defined through the wave vector of the density modulation in the smectic phases ( $\mathrm{Sm} \mathrm{A}$ or $\mathrm{Sm} \mathrm{C}$ ).

Experimental situations [2,3] of NAC point are known and a comparison with the predictions of Chen and Lubensky can be made. There is a general agreement with the predicted topology of the phase diagram but some discrepancies exist. The N-Sm C transition, which is predicted to be of second order is found to be always of first order, and the NAC point is also a tricritical point. It was shown also ( $F$. Hardouin, private communication) that similar phase diagram with NAC point exists with cholesteric molecules and the structure of the smectic $\mathrm{C}$ phase is helicoidal. In such a case, the model of Chen and Lubensky cannot be used and the concept of an order parameter identified with the tilt angle is very useful [4].

The discrepancy concerning the first order character of the transition $\mathrm{N}-\mathrm{Sm} \mathrm{C}$ has been investigated by Swift [5]. He has shown that if the director fluc-

(*) On sabbatical leave from the Technion-Israel, Institute of Technology, Haifa Israel. tuations particular to the $\mathrm{Sm} \mathrm{C}$ ordering are taken into account, the transition N-Sm C is always of first order. However, since his theory is based on approximation, it is not clear whether the first order transition he found is an intrinsic property of the model or it is merely introduced by the approximation itself. It can be shown [6] that using some approximations (like the Hartree approximation) spurious first order transition appears, only because the nature of the approximation used in the calculation.

Thus, it seems justified to find an another approach. In reference [2], a Landau theory with two order parameters has been presented. The first order parameter $\psi$ is characteristic of the appearance of layers in the smectic phases. The second one, $\varphi$ can be identified with the tilt angle. In the nematic phase $\psi$ and $\varphi$ are null together (disordered phase). But the free energy $G$ proposed in reference [2] has a serious flaw. In the nematic phase, one of the stability conditions is $\left(\partial^{2} G / \partial \varphi^{2}\right)_{\psi=0, \varphi=0}>0$, but with the free energy of Johnson et al. [2], we have always $\left(\partial^{2} G / \partial \varphi^{2}\right)=0$ if $\psi=0$, whatever the values of $\varphi$. This means that the surface $G(\psi, \varphi)$ has very particular topological properties and that the fluctuations of $\varphi$ in the nematic phase are very large, while the fluctuations of $\psi$ are small ! Furthermore, these authors found that the N-Sm C transition is of first order because the coefficient of $\psi^{4}$ in the free energy expansion becomes null precisely at the NAC point. It is an ad hoc explanation which is not very satisfactorily. 
In this paper, we present also a Landau theory but with a large advantage since we are able to offer a simple explanation to the first order nature of the $\mathrm{N}$-Sm C transition.

2. Transition with two order parameters. - It was proved [7] that if a transition occurs directly from a disordered state $(\psi=0, \varphi=0)$ to a bi-ordered state $(\psi \neq 0, \varphi \neq 0)$ the transition is, in general, of first order. We recall briefly the demonstration. Let be $G(\psi, \varphi)$ the free energy. The functions $\psi(T)$ and $\varphi(T)$ are calculated from the conditions $(\partial G / \partial \psi)=0$ and $(\partial G / \partial \psi)=0$ or

$$
g_{1}(\psi, \varphi, T)=0 \quad \text { and } \quad g_{2}(\psi, \varphi, T)=0 .
$$

A transition is of second order if $\psi$ and $\varphi$ are null together for $T=T_{\mathrm{c}}$. The eq. (1) becomes

$$
g_{1}\left(0,0, T_{\mathrm{c}}\right)=0 \text { and } g_{2}\left(0,0, T_{\mathrm{c}}\right)=0 .
$$

This gives two equations to determine $T_{\mathrm{c}}$. In general these is no solution and the direct transition

$$
(\psi=0, \varphi=0) \rightarrow(\psi \neq 0, \varphi \neq 0)
$$

cannot be of second order, unless for particular values of the parameters appearing in the expression of $G$. We can also have a gradual ordering such as (for example)

$$
(\psi=0, \varphi=0) \rightarrow(\psi \neq 0, \varphi=0) \rightarrow(\psi \neq 0, \varphi \neq 0)
$$

The results of Imry [8] are in agreement with our conclusions. He used the following form of the free energy

$$
G=\alpha \psi^{2}+\frac{1}{2} \beta \psi^{4}+A \varphi^{2}+\frac{B}{2} \varphi^{4}+b \varphi^{2} \psi^{2}
$$

with $\alpha$ and $A$ linearly temperature dependent. He got that a direct transition from the disordered state to the bi-ordered state takes place through a second order transition only if $\alpha$ and $A$ become zero at the same temperature. Otherwise, there is only a gradual ordering.

3. Phase diagram around a NAC point. - The results of Imry have shown that we have to take more terms in the free energy expansion. There we shall use the following form of the free energy :

$$
\begin{aligned}
G=\alpha \psi^{2}+\frac{1}{2} \beta \psi^{4}+ & \frac{1}{3} \gamma \psi^{6}+ \\
& +A \varphi^{2}+\frac{B}{2} \varphi^{4}+b \varphi^{2} \psi^{2} .
\end{aligned}
$$

As mentionned above $\psi$ is the order parameter of the smectic ordering (first Fourier component of the spatial density variation) and $\varphi$ is the tilt angle of the smectic $\mathrm{C}$ phase. Since the tilt angle is non zero only if $\psi$ is already non zero, we have to suppose that only $\alpha$ can change its sign. We take $\beta$ and $\gamma$ positive in order to have always a second order N-Sm A transition, as found experimentally, in the vicinity of the NAC point. Since, we do not want to get the solution $\psi=0, \varphi \neq 0, A$ and $B$ are taken always positive. Finally, we have to choose $b<0$, in order that the solution $(\psi \neq 0, \varphi \neq 0)$ will be possible. If $\varphi=0$, (3) reduces to

$$
G_{\psi}=\alpha \psi^{2}+\frac{1}{2} \beta \psi^{2}+\frac{1}{3} \gamma \psi^{6}
$$

and if $\alpha>0$ the Nematic phase is stable and if $\alpha<0$ the smectic A phase may be stable.

If $\varphi$ is not identically null, one can write $G$ as a function of $\psi$ only, after calculating $\varphi$ through $\partial G / \partial \varphi=0$. We have

$$
\begin{aligned}
G_{\psi \varphi}=-\frac{A^{2}}{2 B}+(\alpha & \left.-\frac{A b}{B}\right) \psi^{2}+ \\
& +\frac{1}{2}\left(\beta-\frac{b^{2}}{B}\right) \psi^{4}+\frac{1}{3} \gamma \psi^{6} .
\end{aligned}
$$

We see that the introduction of the sixth order term $\frac{1}{3} \gamma \psi^{6}$ is necessary since $\left(\beta-b^{2} / B\right)$ can be either positive or negative.

The phase diagram is determined by varying the two quantities $\alpha$ and $\beta^{\prime}=\beta-b^{2} / B$ since the appearance of the different phases is related to the conditions $\alpha>0$ or $\alpha<0$ and $\beta^{\prime}<0$ or $\beta^{\prime}>0$.

The resolution is made numerically calculating the free energy in the different phases from the quantities $\psi(T)$ and $\varphi(T)$ (through the condition $\partial G / \partial \varphi=0$ and $\partial G / \partial \psi=0)$ taking into account the different stability condition

$$
\partial^{2} G / \partial \varphi^{2}>0, \quad \partial^{2} G / \partial \psi^{2}>0
$$

and

$$
\frac{\partial^{2} G}{\partial \psi^{2}} \frac{\partial^{2} G}{\partial \varphi^{2}}-\left(\frac{\partial^{2} G}{\partial \psi^{2} \partial \varphi^{2}}\right)^{2}>0
$$

We present the results for two cases, to investigate the influence of the parameter $\gamma$.

1. $\beta=10, \gamma=100$ and $A|b| / B=0.1$.

2. $\beta=10, \gamma=10$ and $A|b| / B=0.1$.

There is a part of arbitrary in choosing these values but we are interested only by the shape of the phase diagram. On figure 1 the phase diagrams are shown, taking $\alpha$ and $\beta^{\prime}=\beta-b^{2} / B$ as parameters. Since we choose $\beta$ and $\gamma$ positive, we get that the N-Sm A transition is always of second order. In particular, we note

- the N-Sm C transition is always of first order as a consequence of the general theorem concerning the direct bi-ordering,

- the Sm A-Sm C transition can be first order or second order, depending of the sign of $\beta^{\prime}:$ if $\beta^{\prime}<0$ both transitions (N-Sm A and Sm A-Sm C) are of 


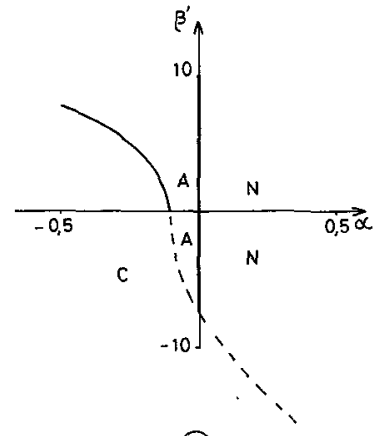

(a)

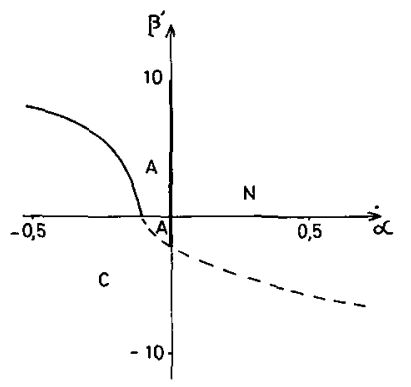

(b)

Fig. 1. - Phase diagram calculated from the free energy with two order parameters. $\beta=10, A|b| / B=0.1: a) \gamma=100, b) \gamma=10$. The thick lines are second order transition lines. The dash lines are those of first order transition. Note the tricritical point at $\beta^{\prime}=0$, on the Sm A-Sm C transition line.

first order. It is interesting to note that the phase diagram is similar to that of Chen and Lubensky, except in the very vicinity of the NAC point.

The tricritical point that we predict on the Sm A-Sm C transition line has not been observed. It is likely that this point is very near the NAC point itself and difficult to observe. The results displayed on figure 1 show that if $\gamma$ is not very large it may be very difficult to observe the tricritical point.

Sigaud et al. [3] have shown that by a linear transformation of the relevent parameters (here $\alpha$ and $\beta^{\prime}$ ) one can get a phase diagram very similar to that which has been experimentally observed. As an illustration, we did the following transformation for the variables $\left(\alpha, \beta^{\prime}\right)$ to the variables $T$ and $x$ (temperature and composition) (for the case $\gamma=100$ )

$$
\begin{aligned}
T & =0.91\left(\beta^{\prime}+10 \alpha\right)+56^{\circ} \mathrm{C} \\
x & =3.22 \times 10^{-2} \beta^{\prime}+0.258 .
\end{aligned}
$$

On figure 2 we see that the new phase diagram is very similar to that observed by Johnson et al. [2]. A better agreement can be obtained if we suppose that the other parameters depend slightly on $T$ and $x$. In particular, the inflection point at the tricritical point on the $\mathrm{Sm}$ A-Sm C line disappears.

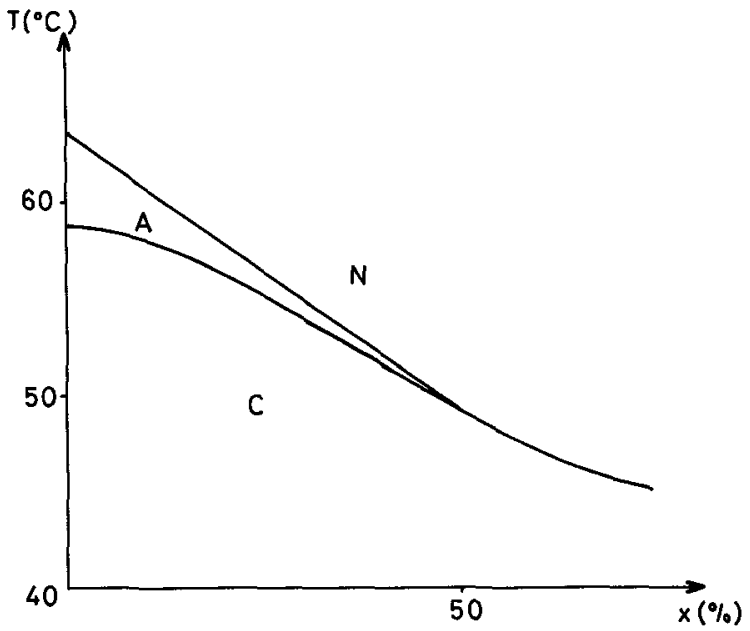

Fig. 2. - Phase diagram constructed from the diagram of the figure $1 a$, by a linear transformation of the variables $\alpha, \beta^{\prime}$ to the variables $x, T$, in order to get a diagram analogous to that of ref. [2].

4. Conclusion. - We have presented a Landau theory of the NAC point, with two order parameters. The results are in good agreement with the experimental observations. In particular, we have a very simple explanation of the first order character of the N-Sm C transition.

\section{References}

[1] Jing-Hue Chen and Lubensky, T. C., Phys. Rev. A 14 (1976) 1202.

[2] Johnson, D., Allender, D., De Hoff, R., Maze, C., OppenHeIM, E. and ReYnolds, R., Phys. Rev. B 16 (1977) 470.

[3] Sigaud, G., Hardouin, F. and Achard, M. F., Solid State Commun. 23 (1977) 35.
[4] Michelson, A., Benguigui, L. and Cabib, D., Phys. Rev. A 16 (1977) 394.

[5] Swift, J., Phys. Rev. A 14 (1976) 2274.

[6] Benguigui, L., Phys. Rev. B 11 (1975) 4547.

[7] Benguigui, L., Phys. Lett. 33A (1970) 79.

[8] IMRY, J., J. Phys. C 8 (1975) 567. 